\title{
UNIVERSAL EMBEDDED RECONFIGURABLE HARDWARE PLATFORM FOR MULTIMEDIA APPLICATIONS IN REAL-TIME
}

\author{
Michael Livshitz, Alexey Petrovsky, Andrey Stankevich, Mikhail Kachinsky, \\ Alexander Petrovsky
}

\author{
Computer Engineering Department, \\ Belarusian State University of Informatics and Radioelectronics, \\ P. Brovky, 6, 220013, Minsk, Belarus, \\ mlivshitz@bsuir.by, petrovsky@bsuir.by, stankevich@bsuir.by, \\ kachinsky@bsuir.by,palex@bsuir.by
}

\begin{abstract}
This paper deals with reconfigurable hardware platform for different purposes real-time speech and audio signal processing. A design conception and turnkey solution are described. Much attention is paid to reconfigurable peripheral processor meant for external interface realization, pre- and post-data processing as well as digital signal processing algorithms implementation with the object of the DSP unloads. Moreover, three applications implemented on the considered platform are demonstrated.
\end{abstract}

Keywords: DSP, FPGA, DISC, Reconfigurable Structure.

\section{INTRODUCTION}

The effectiveness of hardware platforms traditionally based on DSP executing user-written program can be significantly increased using FPGA co-processor. At the same time, increasing of effectiveness is achieved by means of inherent FPGA flexibility that provides implementation of not suitable operations for DSP freeing last one for data processing task. Loading these instructions into FPGA and leaving target instructions that need highspeed processing for DSP performance and cost of the DSP platform are optimized. Moreover, DSP platform that includes FPGA co-processor can easily be reorganized to meet the requirements of the current task by algorithms distribution among DSP and FPGA.

FPGA co-processor can realize function of peripheral processor, implementing hardware platform interface with specific external equipment in audio signal processing tasks.

\section{HARDWARE PLATFORM DESIGN CONCEPTION}

The Core Processor (See Fig. 1) presents a static component of the system, the main task of which is control: managing $\mathrm{I} / \mathrm{O}$ operations, initiating of application-specific instructions, and providing the reconfiguration sequences in so-called Instruction Space.
The latter component is a dynamic part of the system usually built on reconfigurable FPGA(s). Configurations implementing the circuitry needed by the DSP algorithm are being loaded into the instruction space. These predefined configurations (instructions from the point of view of the Core Processor) individually respond to the initiation signals that depend on the DSP algorithm that being implemented by the processor.

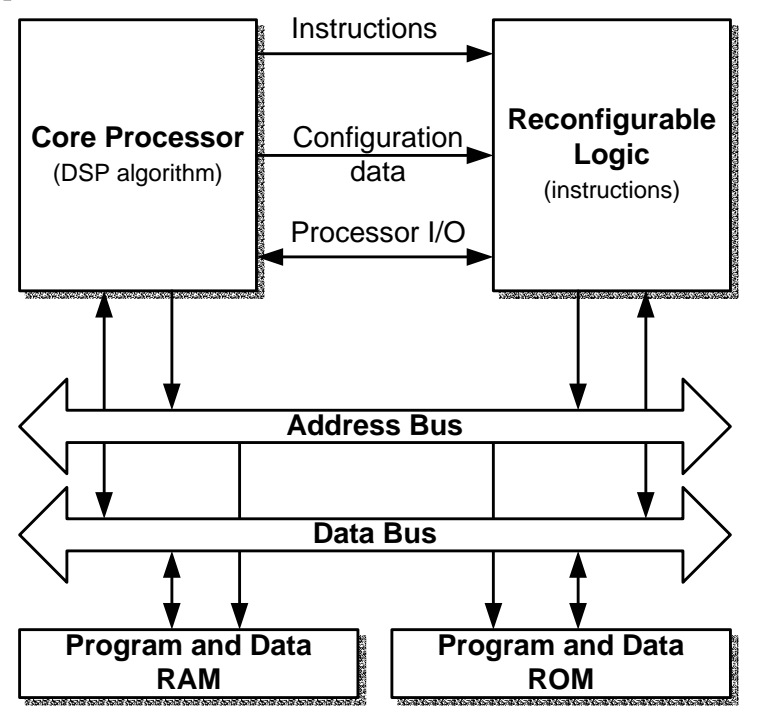

Fig. 1 - Reconfigurable processor structure

Taking into account the increasing abilities of modern FPGA for real-time reconfiguration, it is 
well justified to use this approach in various DSP applications by using specific circuitry libraries that boost the performance of the currently used algorithm. At the same time, the structure of the reconfigurable processor remains unchanged, that yields a significant flexibility particularly suited for embedded processors.

\section{SPECIFICATION}

The proposed hardware platform is based on a dual-core processor consisting from TI TMS320C6713 DSP [1] and FPGA Xilinx Spartan-3 XC3S200 [2] realizing peripheral processor functions. DSP provides executing complex algorithms of digital processing of audio signals.

The peripheral processor meant for hardware platform external interface realization, pre- and postdata processing, supplying with necessary interfaces data formats, external interface packets forming and disassembling as well as digital signal processing algorithms implementation with the object of DSP unload.

The DSP TMS320C6713 is a highestperformance floating-point digital signal processor (DSP) based on Advanced Very Long Instruction Word (VLIW) architecture: Eight 32-Bit Instructions/Cycle; 32/64-Bit Data Word; 200-MHz (PYP) Clock Rates; 3.3-, 4.4-, 5-, 6-Instruction Cycle Times; 1800/1350 MIPS/MFLOPS; rich peripheral set optimized for audio; highly optimized $\mathrm{C} / \mathrm{C}++$ compiler; extended temperature devices available.

The major features of DSP are following:

Eight Independent Functional Units: 2 ALUs (Fixed-Point); 4 ALUs (Floating-/Fixed-Point); 2 Multipliers (Floating-/Fixed-Point); Load-Store Architecture With 32 32-Bit;

General-Purpose Registers: Instruction Packing Reduces Code Size; All Instructions Conditional.

Instruction Set Features: Native Instructions for IEEE 754; Single- and Double-Precision; ByteAddressable (8-, 16-, 32-Bit Data); 8-Bit Overflow Protection; Saturation; Bit-Field Extract, Set, Clear; Bit-Counting; Normalization.

L1/L2 Memory Architecture: 4K-Byte L1P Program Cache (Direct-Mapped); 4K-Byte L1D Data Cache (2-Way); 256K-Byte L2 Memory Total: 64K-Byte; L2 Unified Cache/Mapped RAM, and 192K-Byte Additional L2 Mapped RAM.

Device Configuration: Boot Mode: HPI, 8-, 16-, 32-Bit ROM Boot; Endianness: Little Endian, Big Endian;

32-Bit External Memory Interface (EMIF) [3]: Glueless Interface to SRAM, EPROM, Flash, SBSRAM, and SDRAM; 512 MB Total Addressable External Memory Space.
The Enhanced Direct-Memory-Access (EDMA) Controller [4] (16 Independent Channels), 16-Bit Host-Port Interface (HPI), two McASPs, two InterIntegrated Circuit Bus (I2C Bus), two Multichannel Buffered Serial Ports, two 32-Bit General-Purpose Timers, Dedicated GPIO Module with 16 pins (External Interrupt Capable), Flexible PhaseLocked-Loop (PLL) Based, Clock Generator Module IEEE-1149.1 (JTAG), Boundary-ScanCompatible, 0.13- $\mu \mathrm{m} / 6$-Level Copper Metal Process, CMOS Technology, $3.3 \mathrm{~V}$ I/Os, $1.2 \mathrm{~V}$ Internal.

The external memory and peripheral processor (FPGA) are connected to the TMS320C6713 through the 32-Bit EMIF. The EMIF has four address spaces CE0-CE3 (see Fig. 2). The SDRAM is mapped on the CE0, FLASH memory - CE1, FPGA - CE2. The SDRAM memory with total capacity of $8 \mathrm{MB}$ is connected to processor via internal controller that is a part of the EMIF and operates on the maximum clock frequency available for the memory of this type. Flash memory with total capacity of $2 \mathrm{MB}$ intended for storing DSP program and constant coefficients that are required by signal processing algorithms. The processor uses flash-memory in boot-mode. The program is automatically loaded into DSP's internal memory and external SDRAM by means of second-order boot loader after module initialization that is prepared by first-order boot-loader. The program load and its debug are realized with the help of PC that wired to the external DSP JTAG interface (see Fig. 3).

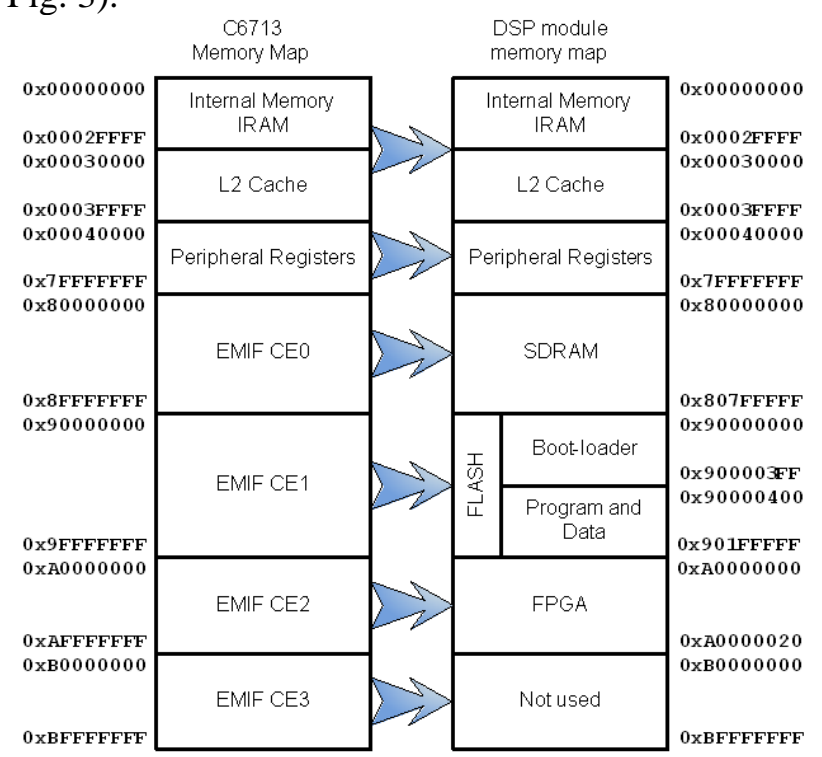

Fig. 2 - DSP Module Memory Map

The TLV320AIC23 codec [5] is used for stereo audio signal input and output purposes. Last one is wired to the DSP via two serial channels: McBSP0 (control channel), McBSP1 (receive/transmit channel). The synchronize clock frequency can be 
adjusted by the Digital Clock Manager (DCM) blocks (FPGA) (see Fig. 3).

For system external interface implementation, FPGA's I/O ports are used (for package PQ208 the number of user outputs are 141). The Spartan-3 family FPGAs supports 26 I/O standards 8 of which are differential (LVDS, LVPECL etc.). In the part of I/O ports, voltage level translators provide connection capability with systems with power level more than $3.3 \mathrm{~V}$. Additional coupling capabilities are provided by Schmitt triggers that allows stable operating with long rising and negative-going slopes signals.

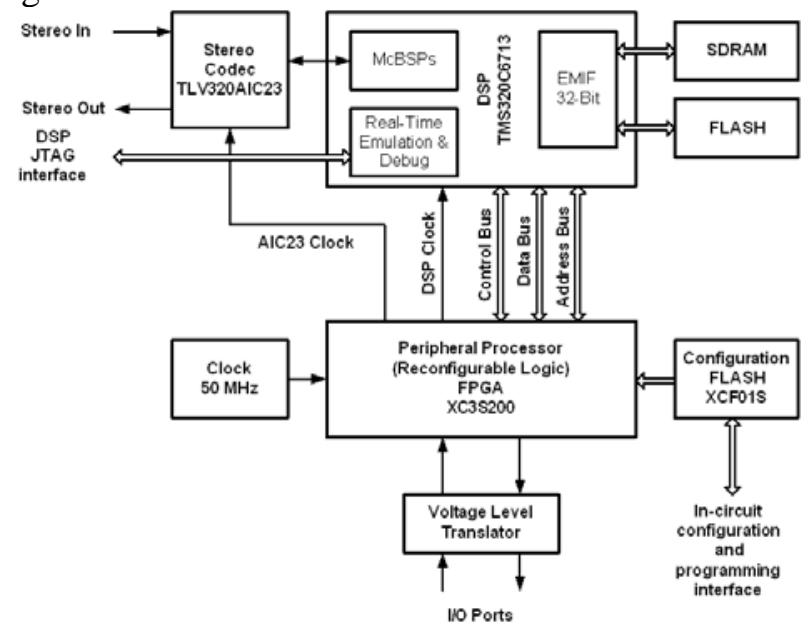

Fig. 3 - Module Structure

The I/O ports can be used as one or several parallel data buses of necessary capacity as well as serial lines. In addition to logic cells, FPGA includes triggers, memory blocks, matrix multipliers and phased-locked loop schemes. Twelve matrix multipliers blocks with 18-bits operands and 36-bits result can be used to implement digital signal processing functions on the peripheral processor.

Realization of peripheral processor project can be done with the help of CAD Xilinx ISE ${ }^{\circledR}$ [6]. In this case, FPGA configuration sequence is generated by means of iMPACT utility and loaded through JTAG interface into the platform's flash-memory. Every time after power up FPGA reads this sequence from flash-memory.

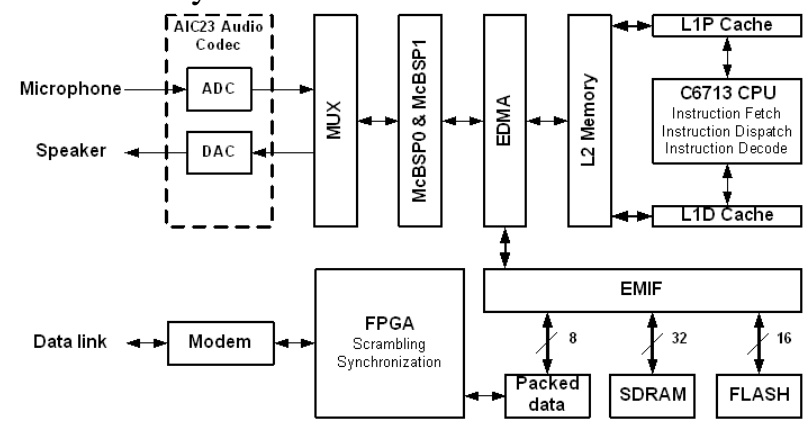

Fig. 4 - Block diagram of the proposed hardware platform
Described architectural decision (see Fig. 4) allows taking following hardware platform features:

1) power computational floating-point core allowing up to 1800/1350 MIPS/MFLOPS;

2) task paralleling possibility in dual-core system;

3) platform reconfiguration via FPGA configuration sequence and DSP-core software change;

4) platform embedding capability into various equipment due to flexible setting of external interface by peripheral processor;

5) standard configuration and programming means [6],[7].

Supply voltage of the module is $+5 \mathrm{~V}$ DC, current consumption is less than $300 \mathrm{~mA}$.

\section{APPLICATIONS}

\subsection{SPEECH ENHANCEMENT SYSTEM}

The complete implementation details of the WDFT based noise reduction system were described in [8]. The block diagram of the WDFT based system is depicted in Fig. 5. In that implementation, a common psychoacoustically motivated spectral weighting technique is used for noise reduction.

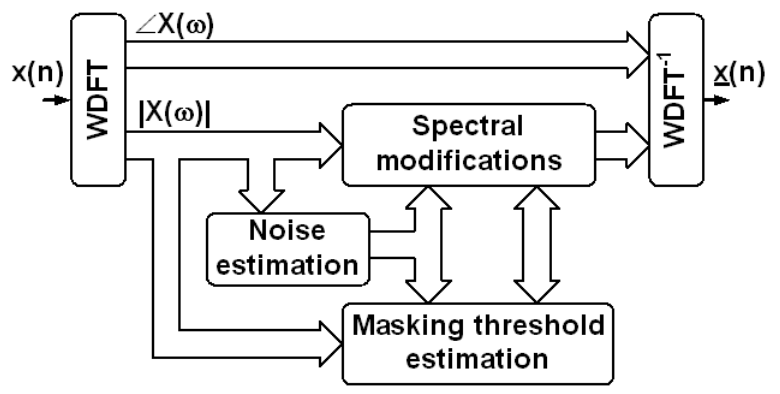

Fig. 5 - Block diagram of the WDFT based perceptual noise reduction system

For experiments was used the set of eight speech sentences with strong high frequency components. The sentences were about a 5-8s long. As a degradation signal, the colored noise was added to the clean speech such that the segmental signal to noise ratio (SEGSNR) was between $-5 \mathrm{~dB}$ and 20 $\mathrm{dB}$. An objective performance evaluation was based on the instrumental and perceptual measurement (see Fig. 6). An example of speech enhancement by means of proposed noise reduction system is depicted in Fig.7. (Car noise at $\mathrm{SNR}=5 \mathrm{~dB}$ was used).

Speech distortions were measured using segmental signal to noise ratio (SEGSNR) where the noise is interpreted as a difference between original and enhanced speech. The higher value of the segmental SNR indicates the weaker speech distortions.

The cepstral distance was also used as a speech distortion measure. The distance between clean and 
enhanced speech cepstra was calculated. In this case, the higher cepstral distance reflects the stronger speech distortions. The amount of noise reduction was measured using noise attenuation factor (NA) which is defined as the mean ratio between the input noise power and output noise power. The instrumental measurement is relatively low correlated with the speech perception. Thus perceptual distortion defined as audible difference between clean and enhanced speech was evaluated using Modified Bark Spectral Distortion (MBSD) measure [9].
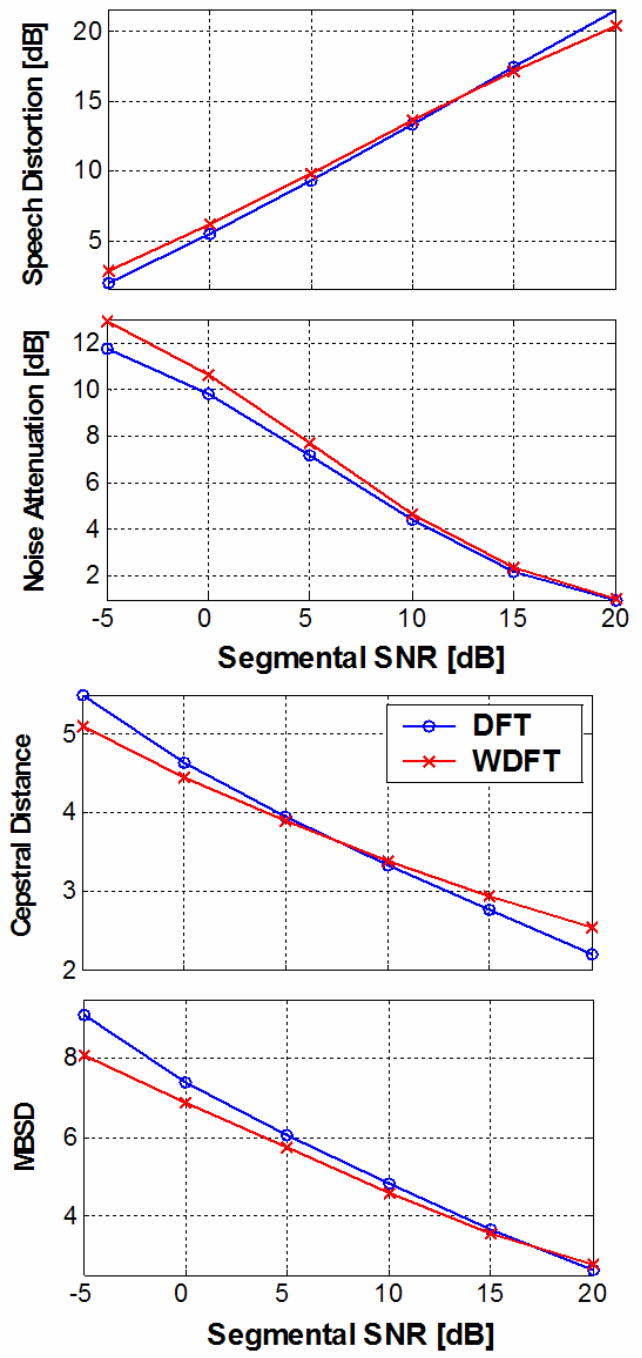

Fig. 6 - Performance evaluation of the WDFT speech enhancement system

The experimental results depicted in Fig. 6 show that proposed approach outperforms both conventional DFT and ordinary WDFT solutions. Extended WDFT system has significantly better noise attenuation performance than conventional DFT system and comparable to pure WDFT method.

Moreover, the spectrograms in Fig.7 testify that proposed noise reduction system is effective enough to produce perceptually clean speech.

\subsection{COMBINED SYSTEM OF PERCEPTUAL WIDEBAND SPEECH CODER WITH NOISE REDUCTION PREPROCESSOR}

The system works in the following way. We used a technique where two buffers (referred to as the PING buffer and the PONG buffer) are used for sampled data transfer by means of EDMA [4] via McBSP port from/to TLV320AIC23 audio codec (See Fig. 4).
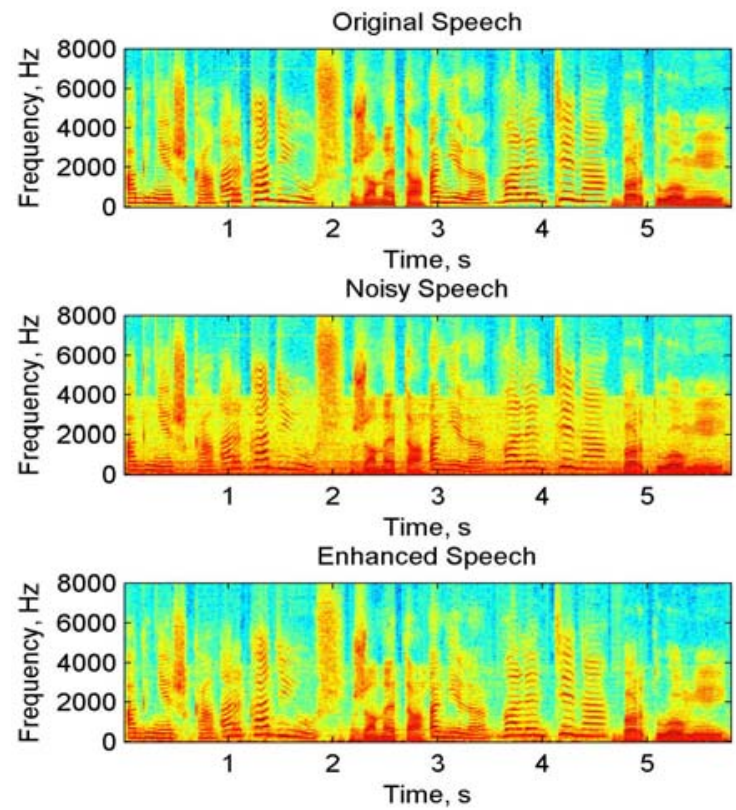

Fig. 7 - WDFT noise reduction system demonstration spectrograms for car noise at $\mathrm{SNR}=5 \mathrm{~dB}$

Input wideband speech $\left(f_{S}=16 \mathrm{kHz}\right)$ goes to the noise reduction unit where clean speech spectrum estimation and masking threshold evaluation is performed. This information is used to estimate spectral weighting coefficients and further processing of noised speech. Using the masking threshold estimates in each bark (twenty one in all for $8 \mathrm{kHz}$ speech bandwidth), subband masking threshold evaluation is implemented by grouping barks according to subband decomposition scheme accepted in the coder [10],[11]. Evaluation of the subband perceptual entropy (SPE) is used by codebook structure monitoring unit with the object of multiband codebook reconfiguration. When the coder's structure is tuned and speech enhancement is done, speech coding process is realized. The combined system is outlined in Fig. 8, the results spectrogram are present on the Fig. 9 and relative MBSD improvement is shown on a Fig. 10.

Noise reduction system partitions input signal frames of length $N=320$ samples (20 ms) with 50\% overlap and multiplies by Hamming window. To 
provide embedding capability we have chosen analysis window size of extended WDFT [12],[13] $N=320$ samples and overcomplete basis size $M=512$.

The noise reduction unit adds a half-frame algorithmic delay $(10 \mathrm{~ms})$ which results in a total algorithmic delay of $30 \mathrm{~ms}$ in combined speech coding system. For the perceptual weighting rule the residual noise level was set to $-26 \mathrm{~dB}$. The LSA rule was configured to achieve maximum noise reduction of $20 \mathrm{~dB}$ and to work with noise at SNR higher than $-10 \mathrm{~dB}$. The factor for decision directed approach was set to $\alpha=0.05$. Encoder operates at variable bitrates (from $4.3 \mathrm{kbps}$ up to $24.2 \mathrm{kbps}$ ) and provides following subjective speech quality estimates: syllabic legibility - 98\%, MOS - 4.2, speaker's voice recognizability $-95 \%$.

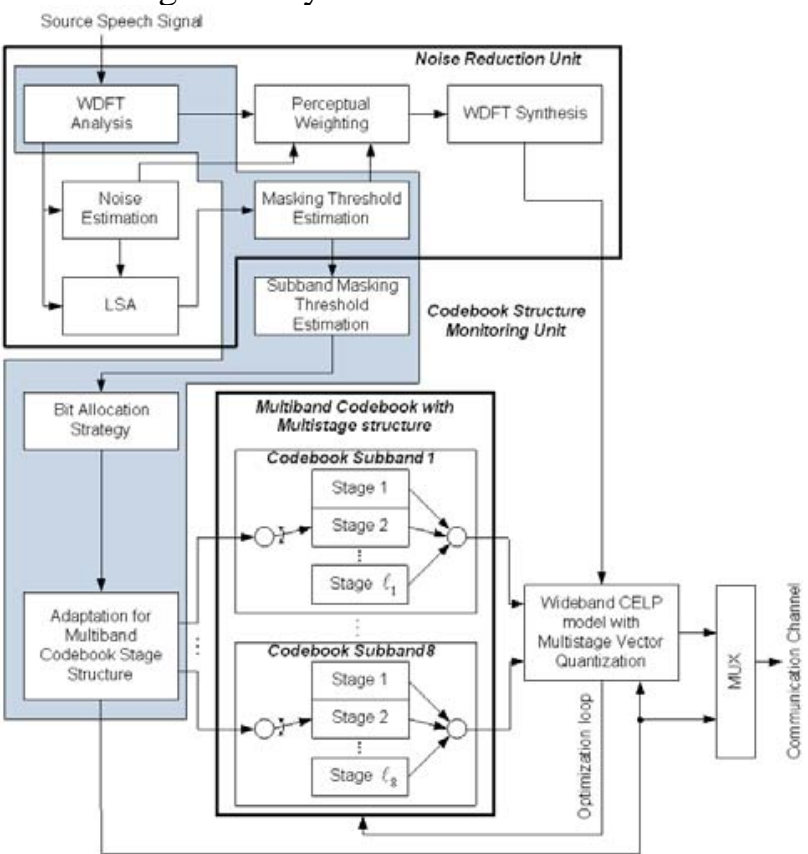

Fig. 8 - Block diagram of combined system and noise reduction system

The objective estimates of the reconstructed speech quality are following: $\mathrm{SNR}=11.59 \mathrm{~dB}$, Noiseto-Mask Ratio (NMR) [14] -6.76dB, BSD $=0.05$, $\mathrm{MBSD}=0.02$. The overall computational complexity distribution among the parts of the encoder system is depicted in Fig. 11.

The memory requirements for combined speech coding system are represented in Table 1.

In the real-time implementation, such complex algorithms and plentiful hardware devices needs to be managed by task manager. For example, in this application four hardware, two software interrupts and three tasks are used (see Fig. 12) [15]. In common case, number of interrupts and background tasks can be different to meet the requirements of an application.
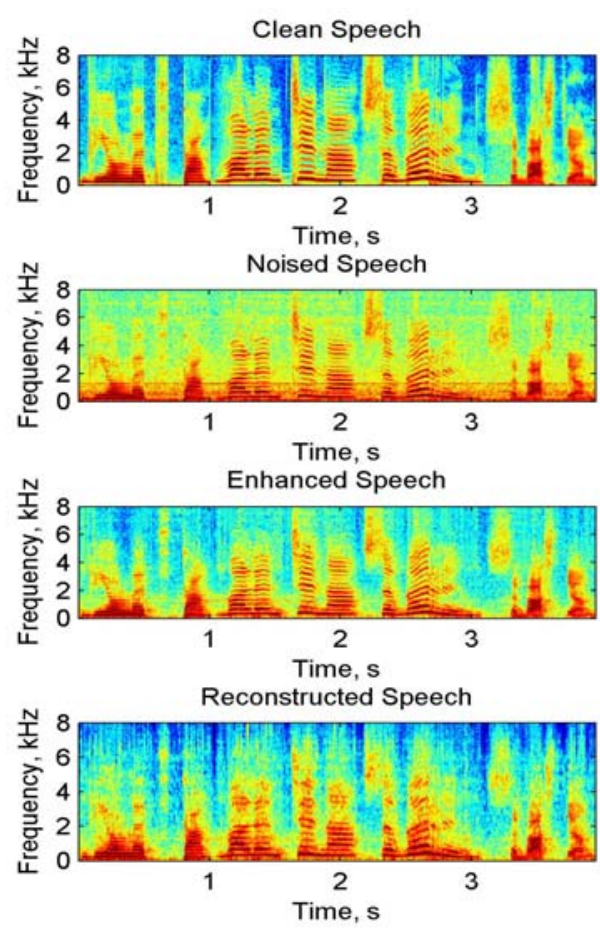

Fig. 9 - Spectrograms of original, noised, enhanced and reconstructed signal

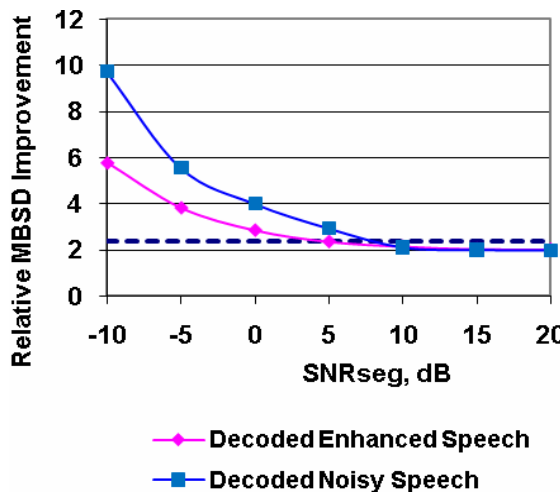

Fig. 10 - Relative MBSD Improvement of noise reduction system

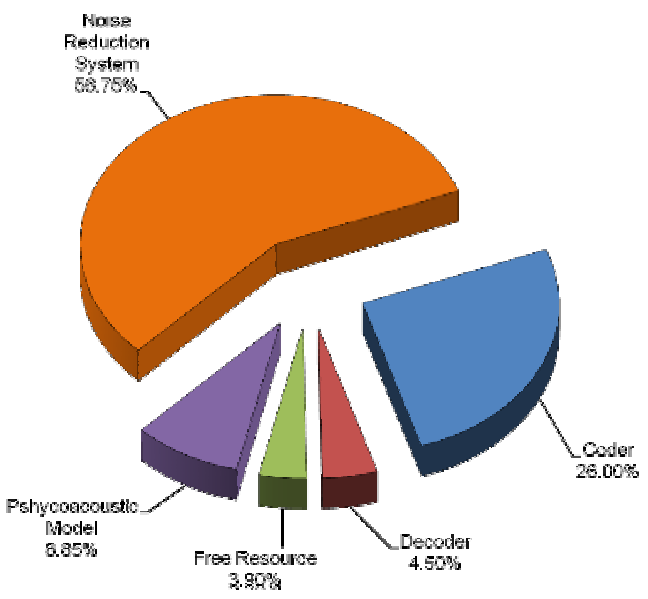

Fig. 11 - Computational complexity distribution 
Table 1. Combined Speech Coding System Memory Requirements

\begin{tabular}{|c|c|c|c|c|}
\hline \multirow{2}{*}{ Memory Type } & \multicolumn{3}{|c|}{ Used Memory, KB } & \multirow{2}{*}{ Available, KB } \\
\cline { 2 - 5 } & Program Memory & Data Memory & Total & 192 \\
\hline IRAM & 178 & 10 & 188 & 8192 \\
\hline SDRAM & 67 & 3156 & 3223 & 2048 \\
\hline
\end{tabular}

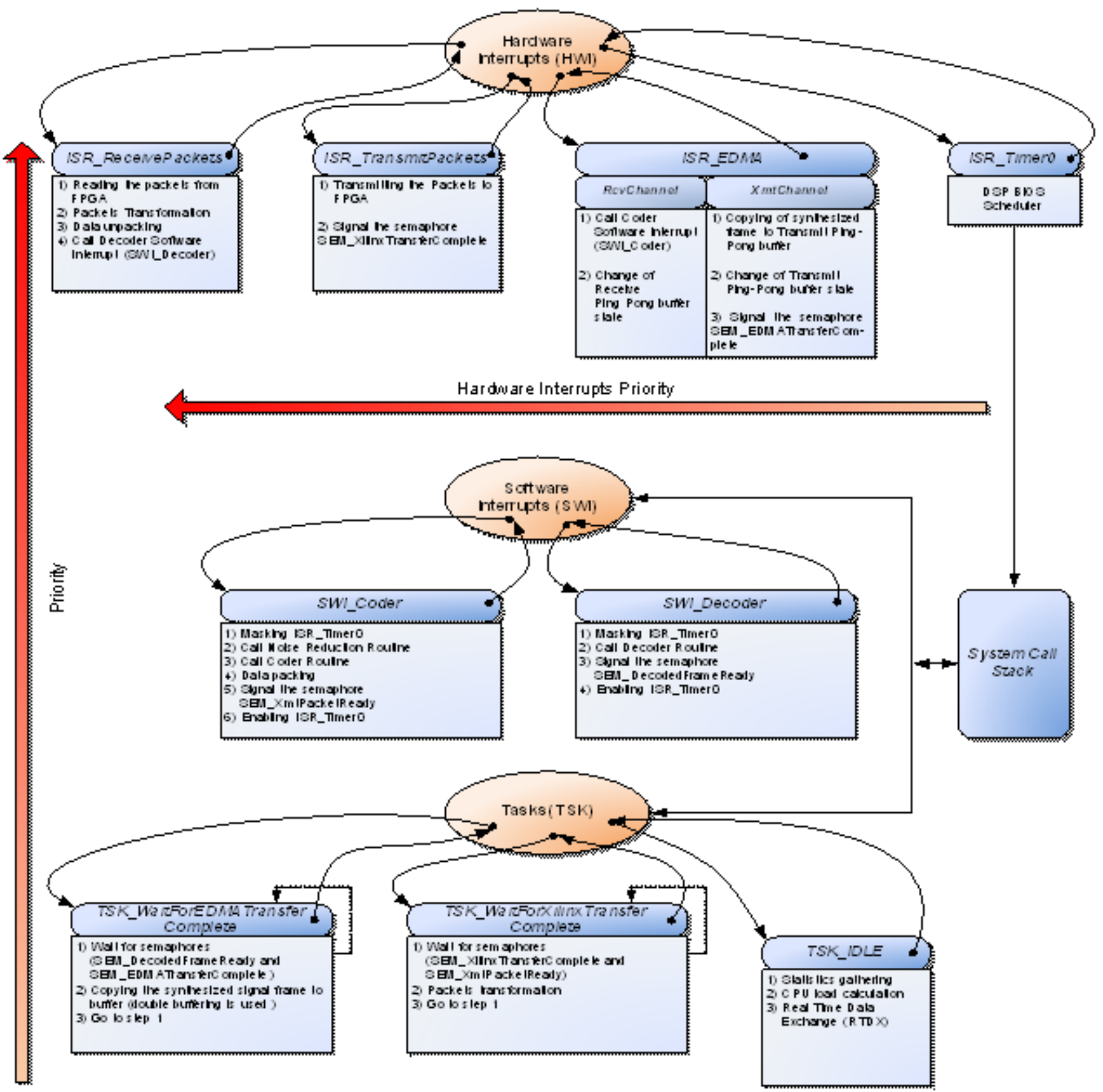

Fig. 12 - Combined speech coding system task managing diagram

\subsection{TRANSFORM AUDIO ENCODER BASED ON THE WDFT}

The encoder and decoder structures for wideband speech and audio signal perceptual coding based on the WDFT are shown in Fig. 13 and Fig 14 [16]. The main components of the coder are forward and inverse WDFT, masking threshold estimation block, quantization/dequantization and lossless Huffman coding/decoding modules.

The performance of the wideband perceptual WDFT speech/audio coder $(16 \mathrm{kHz}$ sampling frequency), defined as the bit-rate, is not greater than 24 kbps. For higher quality audio coding $(32 \mathrm{kHz}$ sampling frequency), it is $46 \mathrm{kbps}$, accordingly. Therefore, the coding gain using adaptive Huffman 
coding for Gaussian modeling is about 1.45 bit per sample for both band signals.

The original and reconstructed spectrograms of wideband perceptual WDFT speech/audio encoding are shown on a Fig 15.

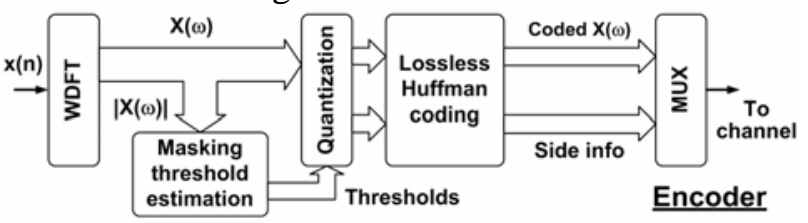

Fig. 13 - The block diagram of the WDFT-based perceptual audio coder

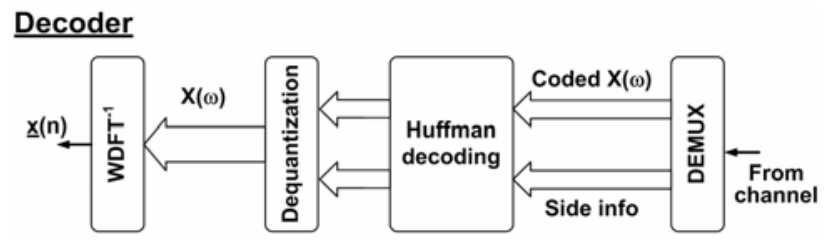

Fig. 14 - The block diagram of the WDFT-based perceptual audio decoder

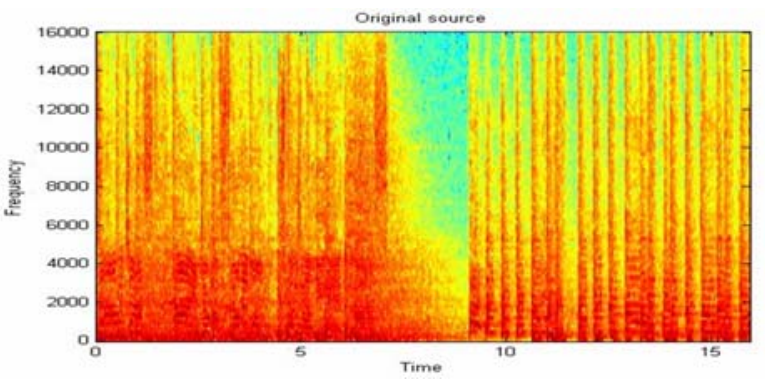

a)

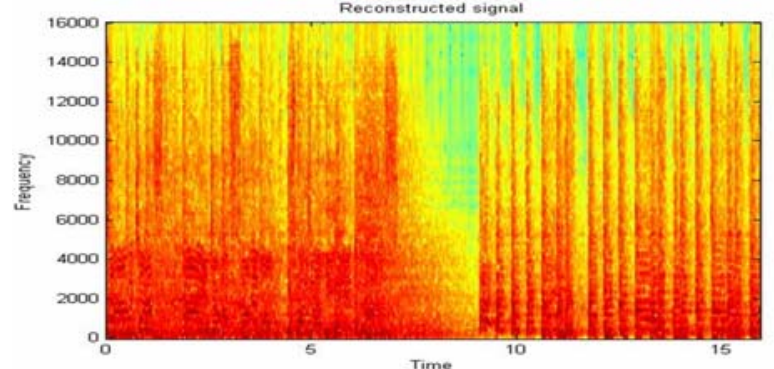

b)

Fig. 15 - Original (a) and reconstructed (b) signal spectrograms

\subsection{FUTURE WORK}

Such a flexible architecture allows designing reconfigurable platforms for different purposes realtime DSP-applications. However, in the future peripheral processor can be implemented with reconfigurable structure of arithmetical and logical units to meet the requirements of the current algorithm. Therefore, overall performance and flexibility of the system can be significantly improved.

\section{REFERENCES}

[1] TMS320C6713B Floating-Point Digital Signal Processor, SPRS294B, Texas Instruments, Dallas, TX, June, 2006. - 152 p.

[2] Spartan-3 FPGA Family: Complete Data Sheet (DS099) [Electronic resource] / Xilinx Inc., May, 2007. - Mode of access: http://direct.xilinx.com/bvdocs /publications/ ds099.pdf. - Date of access: 25.05.2007.

[3] TMS320C6000 DSP External Memory Interface (EMIF) Reference Guide, SPRU266E, Texas Instruments, Dallas, TX, February, 2006. $-146 \mathrm{p}$.

[4] TMS320C6000 DSP Enhanced Direct Memory Access (EDMA) Controller Reference Guide, SPRU234B, Texas Instruments, Dallas, TX, March, 2005. - $269 \mathrm{p}$.

[5] TLV320AIC23B Stereo Audio Codec, Data Manual, SLWS106H, Texas Instruments, Dallas, TX, February, 2004. - 56 p.

[6] ISE Quick Start Tutorial, [Electronic resource] / Xilinx Inc., May, 2007. - Mode of access: http://www.xilinx.com/support/ sw_manuals/ xilinx7/download/qst.pdf. - Date of access: 25.05.2007.

[7] Composer Studio User's Guide, SPRU328B, Texas Instruments, Dallas, TX, 2000.

[8] A.Petrovsky, M.Parfieniuk, A. Borowicz. Warped DFT Based Perceptual Noise Reduction System // Proc. AES 116 ${ }^{\text {th }}$, Berlin, Germany, 8-11 May 2004, Conv. Paper \#6035.

[9] Yang W., Benbouchta M., Yantorno R. Performance of a Modified Bark Spectral Distortion Measure as an Objective Speech Quality Measure // Proc. of ICASSP, Seattle, USA, 1998, pp. 541-544.

[10] M.Z. Livshitz, M. Parfeniuk, A.A. Petrovsky. Wideband CELP-coder with Wideband Excitation and Multilevel Vector Quantization on Code Book with Reconfigurable Architecture // Digital Signal Processing, No. 2, Moscow, 2005, pp. 20-35. (in Russian)

[11] Michael Livshitz and Alexander Petrovsky, Perceptually Constrained Variable Bit Rate Wideband Speech Coder // Proc. of EUROCON, Serbia \& Montenegro, Belgrade, November 22-24, 2005, pp.1296-1299.

[12] Michael Livshitz and Alexander Petrovsky, An Overcomplete WDFT-based Perceptually Constrained Variable Bit Rate Wideband Speech Coder with Embedded Noise Reduction System // Proc. of $11^{\text {th }}$ Int. conf. SPECOM, St. Petersburg, Russia, June 25-29, 2006, pp.343348.

[13] Borowicz A., Petrovsky A., An Overcomplete WDFT Sinusoidal Basis for Perceptually 
Motivated Speech Enhancement // Proc. of 13th EUSIPCO'2005, (CD-ROM), Antalya, 2005.

[14] Brandenburg K., Sporer T. NMR and Masking Flag: Evaluation of Quality Using Perceptual Criteria // The 11th AES Proceedings, Portland, Oregon, USA, May 1992, pp. 169-179.

[15] TMS320C6000 DSP/BIOS Application Programming Interface (API) Reference Guide, SPRU403F, Texas Instruments, Dallas, TX, 2003.

[16] Petrovsky A., Borowicz A., Parfieniuk M., Petrovsky Al. Warped Discrete Fourier Transform in Perceptual Speech and Audio Processing // $X$ Symposium New Trends in Audio and Video, Wroclaw, Poland, 16-18 September 2004 (Prace Naukowe Instytutu telekomunikacji i akustyki Politechniki Wroclawskiej, 85, seria: konferencje 29). pp. 143-152.

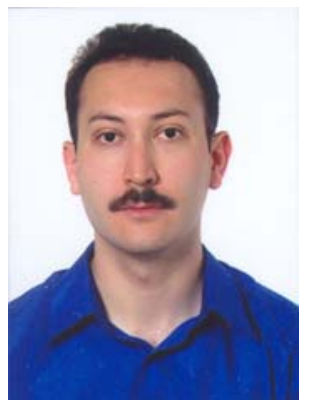

Michael Livshitz received his Dipl.-Eng. degree and Ph.D. in computer engineering from Belarusian State University of Informatics and Radioelectronics (BSUIR) in 2003 and 2007 correspondingly.

He is currently an Associate professor in the Computer Engineering Department of BSUIR since 2007.

He specialized in developing of reconfigurable hardware platforms for multimedia systems. His primary interests are in field of computer device development, digital signal processing, wideband speech and audio coding, voice conversion.

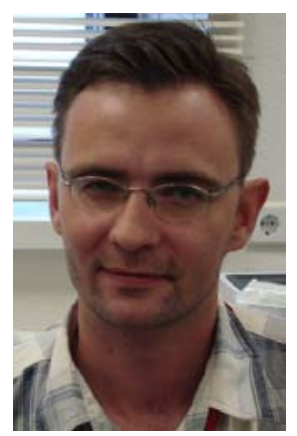

Alexey Petrovsky received his Dipl.-Eng. in computer engineering and Ph.D. in computer science from Belarusian State University of Informatics and Radioelectonics (BSUIR) in July 1998 and October 2001 correspondingly.

$\mathrm{He}$ is currently an Associate professor in the Computer science department of BSUIR since 2001.

$\mathrm{He}$ specialized in developing of reconfigurable hardware platforms for audio processing purpose. His primary interests are in wideband speech and audio processing, perceptual coding and psychoacoustics.

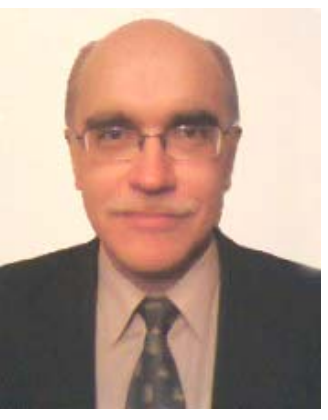

Andrey Stankevich received Dipl.-Eng. degree in computer science from Minsk Radio-Engineering Institute in July 1980. From 1982 to 1992, he was joined with National Academy of Science in Institute of Applied Physics, there he received Ph.D. in electrical engineering in 1988.

He is currently an Associate Professor in the Computer engineering department of Belarusian State University of Informatics and Radioelectronics since 1995.

His primary interests are in a field of computer device development, digital signal processing and technical system modeling.

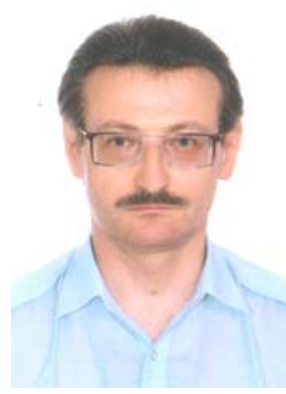

Mikhail Kachinsky received Dipl.-Eng. degree in computer science in July 1980 and Ph.D. in computer engineering in 1990 from Minsk Radio-Engineering Institute.

He is currently an Associate Professor in the Computer engineering department of Belarusian State University of Informatics and Radioelectronics since 1992.

His primary interests are in a field of digital signal processing, high-speed microprocessor and software development for real-time applications.

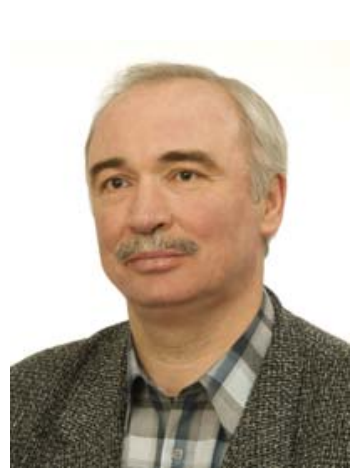

Alexander Petrovsky received the Dipl.-Eng. degree in computer engineering in 1975 and the Ph.D. degree in 1980 both from the Minsk Radio-Engineering Institute, Belarus, USSR. In 1989, he received the Doctor of Science degree from The Institute of Simulation Problems in Power Engineering, Academy of Science, Kiev, Ukraine, USSR. In 1975, he joined Minsk Radio-Engineering Institute. He became a Research Worker and Assistant Professor and since 1980 he has been an Associate-Professor of the Computer Science department. From 1983 to1984, he was a Research Worker at Royal Holloway 
College and Imperial College of Science and Technology, University of London (UK). Since May1990, he has been Professor and Head of the Computer Engineering department, the Belarusian State University of Informatics and Radioelectronics.

Recently his main research interests are acoustic signal processing, such as speech and audio coding, noise reduction and acoustic echo cancellation, robust speech recognition, and real-time signal processing. A.A.Petrovsky is a member of the Russian A.S.Popov Society for Radioengineering, Electronics and Communications, the Editorial Staff Member of the Russian journals Digital signal processing, Speech technology, AES, IEEE, EURASIP. 